\title{
A recurrent mutation in bone morphogenetic proteins-2-inducible kinase gene is associated with developmental dysplasia of the hip
}

\author{
LIHUA ZHAO $^{1 *}$, ZAIWEI ZHOU ${ }^{1 *}$, SUN WANG $^{1}$, QING JIAO $^{1}$, JING WU $^{2}$, \\ FENG MA ${ }^{1}$, LINGYAN FAN ${ }^{1}$, MENGJIE CHEN $^{1}$ and HAO YING ${ }^{1}$ \\ ${ }^{1}$ Department of Orthopedics, Shanghai Children's Hospital, Shanghai Jiao Tong University, Shanghai 200062; \\ ${ }^{2}$ Laboratory of Translational Research, Institute of Pediatric Translational Medicine, Shanghai Children's \\ Medical Center, School of Medicine, Shanghai Jiao Tong University, Shanghai 200071, P.R. China
}

Received December 15, 2015; Accepted December 23, 2016

DOI: $10.3892 / \mathrm{etm} .2017 .4191$

\begin{abstract}
Developmental dysplasia of the hip (DDH) is a complex disorder of the hip joint affecting 1-5\% of newborns. While genetic influence on DDH has been long known, DDH has not been ascribed to any specific genetic event. The present study reported on variants contributing to DDH susceptibility in a family with four individuals affected across three generations. Whole-exome sequencing was performed in three affected and two unaffected individuals of a pedigree with DDH. Candidate variants were confirmed by Sanger sequencing and then validated in available family members and 37 sporadic DDH patients. Two novel heterozygous, inframe mutations causing multi-nucleotide substitution polymorphisms (c.1432_1440delCAGCAGCAG corresponding with p.Gln478_480del and c.1440_1441insCAG corresponding with p.Gln480ins) in exon 11 of chromosome 4 in bone morphogenetic proteins-2-inducible kinase (BMP2K) were identified; these were found in members of the pedigree affected by DDH and in the unaffected grandmother of the proband, who was deemed to be the carrier of potential mutations, but not in the unaffected normal control saunt of the proband. These two variants shared the same genomic coordinate but with different types of mutation in BMP2K. BMP2K is known to be associated with bone and cartridge development and heterozygous mutations were found to be present in $4 / 4(100 \%)$ of the affected family members, $4 / 15(26.7 \%)$
\end{abstract}

Correspondence to: Professor Hao Ying, Department of Orthopedics, Shanghai Children's Hospital, Shanghai Jiao Tong University, 355 Lane Luding Road, Putuo, Shanghai 200062, P.R. China

E-mail: zhaolh0803@163.com

*Contributed equally

Abbreviations: BMP2K, bone morphogenetic proteins-2-inducible kinase; DDH, developmental dysplasia of the hip; WES, whole-exome sequencing; NHLBI, National Heart, Lung and Blood Institute; MNP, multinucleotide substitution polymorphism

Key words: developmental dysplasia of the hip, whole-exome sequencing, susceptibility-inducing mutation of the unaffected family members and 0/7 (0\%) of the unaffected unrelated family members. Genotyping of 37 unrelated, sporadic DDH patients showed that three cases were positive for the BMP2K c.1432_1440delCAGCAGCAG variants (8.12\%). These findings provided strong evidence for the role of BMP2K variants in causing DDH and demonstrated that the combination of pedigree information and next-generation sequencing is an effective method for identifying pathogenic sites associated with DDH.

\section{Introduction}

Developmental dysplasia of the hip (DDH; Online Mendelian Inheritance in Man entry no. 142700) is a spectrum of disorders affecting the proximal femur and acetabulum that leads to hip subluxation or dislocation and suboptimal joint function. One to five of 1,000 newborns are affected by DDH in China (1). Early diagnosis and treatment are important, as failure to diagnose DDH in neonates and young infants may result in significant morbidity, such as early accelerated wear of the articular cartilage resulting in $40 \%$ osteoarthritis of the hip at age 20-40 (2). At present, a sensitive and specific genetic test to accurately identify newborns susceptible for DDH is an elusive goal for pediatric orthopedic surgeons.

DDH is a common, complex, osteologic condition with environmental as well as genetic factors contributing to the risk. Epidemiologic studies have highlighted the significant influence of the orientation of the fetus in the womb on DDH, and Breech presentation (specifically if delivered naturally), primiparity and high birth weight are significant environmental risk factors (3). Genetic contributions are apparent from studies on pedigrees with a 12-fold increase of DDH among first-degree offspring of those affected (4). Evidence for a genetic cause of DDH has been provided by studies indicating a genetic predisposition to DDH based on polygenic inheritance $(5,6)$. Furthermore, monozygotic twins have a $41 \%$ concordance rate as compared to the $2.8 \%$ seen in dizygotic twins (7). At present, few loci and genes have been identified to be associated with DDH. Genome-wide scans of affected families have suggested linkage to chromosomes 4q35 (8), 16p (9), $13 q 22$ (10) and 17q21 (11). To date, no mutations have been found to be definitely linked to DDH in large canine pedigree 
studies, in spite of certain dog pedigrees being commonly affected by DDH (12). Linkage to chromosome 12q13, where the genes encoding collagen type II, alpha 1 (COL2A1) and vitamin $\mathrm{D}$ receptor are located, were recently excluded, as were two polymorphic sites within the COL1A1 gene (13). A recent linkage analysis and a whole-exome sequencing (WES) study in a 71-member family pedigree revealed that a rs3732378 variant in CX3 chemokine receptor on chromosome 3p22.2 was shared by all affected family members and by $15 \%$ of sporadic DDH cases, but it was also carried by some unrelated, 'married-in' individuals in this family $(14,15)$. To date, no gene mutations in these reported regions have been found to be definitely linked to DDH. Indeed, it is the complex genetic heterogeneity that has previously posed a significant barrier to the investigation and molecular diagnosis of DDH.

Through WES, the present study identified for the first time, to the best of our knowledge, that recurrent germline mutations and somatic deletion or insertion in bone morphogenetic proteins-2-inducible kinase (BMP2K) correlated with the phenotype of DDH in a 37-member, three-generation pedigree. The present study provided further evidence that DDH is a genetically heterogeneous disease and that BMP2K mutations account for a subset of the cases.

\section{Materials and methods}

Subjects and clinical analysis. A four-generation, 37-member family from Southern Suzhou (Anhui, China) of Han Chinese origin, in which DDH was present in three generations, and 37 sporadic DDH patients from China were recruited for the present study. The Institutional Ethical Review Board of the Shanghai Children's Hospital (Shanghai, China) approved the protocol of the present study. After obtaining written informed consent from all participants or their legal guardians, family members and sporadic DDH patients were diagnosed using detailed clinical exams and supine anterior posterior radiographs of the pelvis. Results of clinical exams and radiograph imaging of the hips were evaluated by three pediatric orthopedic surgeons, with clinical opinions of two additional surgeons elicited in any case of disagreement. Radiographic measurements of the hip were taken and affected individuals were identified according to the following criteria: Perkin quadrant (the femoral head is not in the inner lower Perkin quadrant), Acetabular index ( $>25$ degrees), Shenton's line (disrupted) and center edge angle ( $<20$ degrees). A pedigree chart was constructed based on the family information (Fig. 1). Four patients had hip dislocation with all the four radiographic signs and were considered to be unequivocally affected by DDH (individuals III3, III10, IV2 and IV6 in Fig. 1). Patient IV6 was the main proband of the present study. A total of 18 individuals had no clinical features or radiographic signs of DDH and were deemed as unaffected, and their blood samples were obtained (individuals marked with asterisks in Fig. 1). Among these, one subject succumbed to lung cancer during the study period (subject II11 in Fig. 1). Three individuals died before the present study was initiated (subjects I1, I2 and II3 in Fig. 1) and 11 refused to donate DNA samples for the present study and were therefore excluded from the analysis. None of the subjects assessed had any systemic syndrome. Peripheral blood samples from affected/unaffected family members and sporadic DDH patients were collected. Genomic DNA was then extracted from the 22 available family members' and 37 sporadic DDH patients' blood samples using the QIAamp DNA Blood Mini kit (cat. no. 51,104; Qiagen, Hilden, Germany) according to manufacturer's protocol. Finally, genetic syndromes involving dysplasia, subluxation or dislocation of the hip and cardiovascular disease were ruled out and confirmed in this family and in the sporadic DDH patients.

Exome and Sanger sequencing. Three affected (III3, III10 and IV6 in Fig. 1) and two unaffected (II7 and III2 in Fig. 1) family members (one was the grandmother of IV6 and was deemed to be a carrier of mutations, and the other one was the aunt of IV6 who did not carry any BMP2K mutation and was used as a control) were selected for WES and for analysis of exonic variants and are shown in the pedigree (Fig. 1). Exome capture on each individual was performed using a SureSelect Human All Exon V5+UTRs (Agilent Technologies, New Castle, DE, USA), guided by the manufacturer's protocols. Sequencing was performed using an Illumina HiSeq 2500 (Illumina, San Diego, CA, USA) following the manufacturer's instructions. A control reference sequence was derived from the 1,000 Genomes project (http://www.1000genomes.org) and from the reference human genome (GRCh37) assembly of the National Center for Biotechnology Information. Exome reads were analyzed in a standard bioinformatics pipeline based on Burrows-Wheeler Aligner for sequence alignment on GRCh37 reference, Broad Institute Genome Analysis Tool Kit (GATKv2.6) for genotyping, ANNOVAR 11 and SnpEff for variant annotation and ExomeDepth for copy number variation detection (16-19). Variants were detected using the GATK Unified Genotyper (software.broadinstitute.org/gatk) in conjunction with Southern Han Chinese and Han Chinese in the Beijing exome BAM files, which is a compressed binary version of a SAM file that is used to represent aligned sequences, from the 1,000 Genomes Project. Potentially deleterious variants were analyzed, which were detected in subjects II7, III3, III10 and IV6 (Fig. 1) but absent in subject III2 (Fig. 1). The single nucleotide polymorphisms, version 137 (dbSNP137) (ncbi.nlm.nih.gov/projects/SNP/), the 1,000 Genomes Project (internationalgenome.org/) and the National Heart, Lung and Blood Institute (NHLBI) databases (nhlbi. nih.gov) were selected for further scrutiny and segregation analysis. Candidate variants were validated in the four affected family members, other available family members as well as in-laws (unrelated individuals married into the family). Whole exons and adjacent splicing site of the candidate gene were tested using Sanger sequencing in 37 sporadic DDH cases. Polymerase chain reaction products were amplified using the primers shown in Table I, which were synthesized by Shanghai Majorbio Co., Ltd. (Shanghai, China). The reaction mixture $(25 \mu \mathrm{l})$ contained $2 \mu \mathrm{l}$ DNA template $(200 \mathrm{ng}), 1 \mu \mathrm{l}$ of each primer $(10 \mu \mathrm{M})$ and $2 \mathrm{X}$ Premix master mix (cat. no. RR820A; Takara Biotechnology Co., Ltd., Dalian, China). PCR thermal cycling was performed as follows: Initial denaturation at $95^{\circ} \mathrm{C}$ for $1 \mathrm{~min}$, followed by 45 cycles of $94^{\circ} \mathrm{C}$ for $10 \mathrm{sec}, 60^{\circ} \mathrm{C}$ for $20 \mathrm{sec}$ and $72^{\circ} \mathrm{C}$ for $25 \mathrm{sec}$. The reaction products were analyzed using the ABI 3730XL Genetic Analyzer equipment (Applied Biosystems; Thermo Fisher Scientific, Inc., Waltham, MA, USA). 


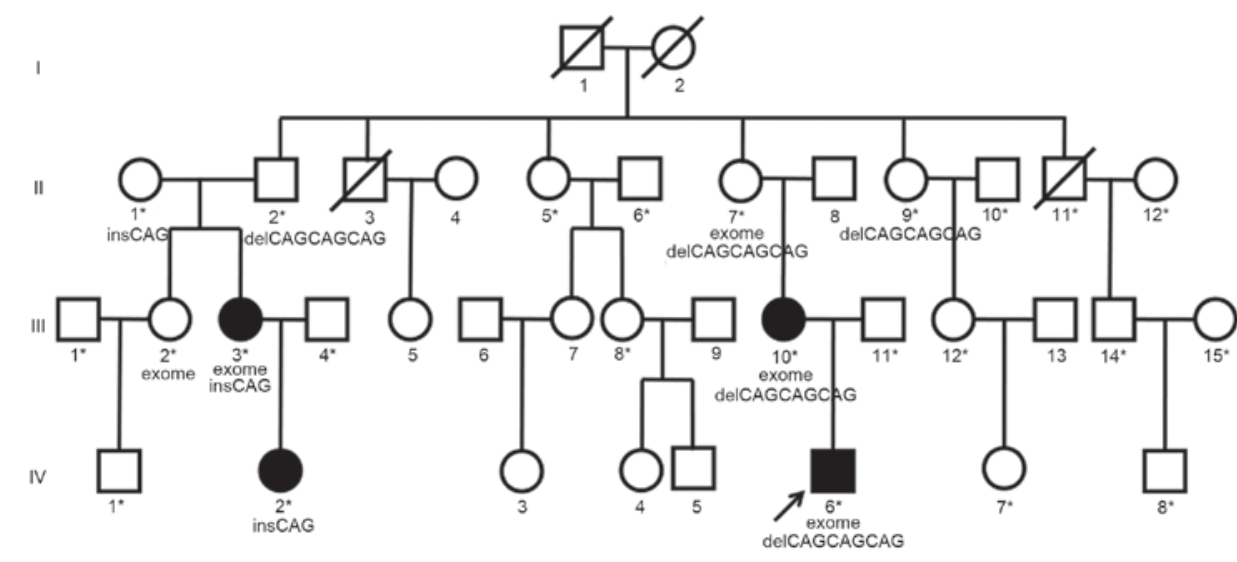

Figure 1. Pedigree assessed in the present study and members of the pedigree carrying BMP2K mutations. Filled in symbols denote individuals with DDH. Symbols with a backslash denote individuals that have died. Asterisks indicate individuals whose DNA samples were retrieved. Five members of the family, which are marked with 'exome', were recruited for whole-exome sequencing analysis. The main proband of the study is indicated by an arrow. 'insCAG' indicates individuals with c.1440_1441insCAG mutation and 'delCAGCAGCAG' indicates individuals with c.1432_1440delCAGCAGCAG mutation. DDH, developmental dysplasia of the hip; Ins, insertion; del, deletion.

\section{Results}

Whole-exome analysis. Whole-exome analysis of the three affected and two unaffected family members (subjects II7, III2, III3, III10 and IV6 in Fig. 1) was performed. An average of 159.74 million 100-bp paired-end sequencing reads were generated for each individual, $98.8 \%$ of which aligned to the targeted region. An average of $96.6 \%$ of targeted regions were covered at a read depth of at least 20x. On average, 38,810 SNPs were identified per individual (range, 36,493-40,633). A total of 168 heterozygote variants (missense/nonsense/indels/inserts), of which $92.8 \%$ were already annotated in a public database (dbSNP v137), found in all three affected individuals and the main proband's grandmother, who was deemed to be the carrier of mutations (individuals II7, III3, III10 and IV6 in Fig. 1), but not found in the aunt of one family member with DDH who was used as a control (subject III2 in Fig. 1). In order to refine the list of variants, a filtering strategy was applied with only those variants included that were indels, those predicted to be loss of function (damaging) mutations by SIFT (sift.jcvi.org/) and ANNOVAR programs (annovar.openbioinformatics.org/) and those that were not included in the dbSNP137, 1,000 Genomes Project and NHLBI databases, and as shown in Fig. 2 (20-22).

After these three filtering steps shown in Fig. 2, two novel heterozygous, inframemutationscausing multi-nucleotidesubstitution polymorphisms (MNPs) (c.1432_1440delCAGCAGCAG corresponding with p.Gln478_480del and c.1440_1441insCAG corresponding with p.Gin480ins) in exon 11 of chromosome $4 q 21.21$ in the BMP2K gene remained, which were possibly correlated with DDH (Fig. 1). These two variants shared the same genomic coordinate but with different types of mutation in BMP2K. The family members affected by DDH and the unaffected grandmother of subject IV6 had either one of these two variants, while the unaffected aunt of another family member had no mutation in BMP2K (Fig. 3A). No shared splice-site mutations or variants were found. The BMP2K variants encode a mutation that caused the deletion of three amino acids or insertion of one amino acid in the BMP2K transcript (NM_017593). This indel was found to affect a evolutionarily conserved glutamine residue within the BMP2K gene.

Table I. Primers used to amplify the sequences harboring the variants in the present study.

Primer name Sequence (5'-3')

Chr4:exon 1-F Chr4:exon 1-R Chr4:exon 2-F Chr4:exon 2-R Chr4:exon 3-F Chr4:exon 3-R Chr4:exon 4-F Chr4:exon 4-R Chr4:exon 5-F Chr4:exon 5-R

Chr4:exon 6-F Chr4:exon 6-R Chr4:exon 7-F Chr4:exon 7-R Chr4:exon 8-F Chr4:exon 8-R Chr4:exon 9-F Chr4:exon 9-R

Chr4:exon 10-F Chr4:exon 10-R Chr4:exon 11-F Chr4:exon 11-R Chr4:exon 12-F Chr4:exon 12-R Chr4:exon 13-F Chr4:exon 13-R

Chr4:exon 14-F

Chr4:exon 14-R

Chr4:exon 15-F

Chr4:exon 15-R

Chr4:exon 16-F Chr4:exon 16-R
TTTCGCTCTCTTCCTCTACCC GCAAGAAAAGCCAGCTTAAGAG GAGAAGCGTCTTCATTCGAGA AGCCAGGTATGGTGATGGAC TCGCATGCAGGTAAACTTTTT TGCGTTTACATGCTCATATCATAA GATGATCTTTTGAGATGTAAAATGTG CTCCACAGCCATGCTAGAAA TTTGACCTTCCTCATAATTTGG AAAATCTGCATCTATGGCAAAA TTCAAGGAAAACTGTTTCCAA AAGCTTTCCATCTCAGGATTG TTCAGCCAACTAGCCACAGA TCAATAATTAGGTTGCTTTCATTCTT CCTTAGCTTGGGATAATGCTT TCCCCAAGGTATCTCAGTGC TGGCACATGAATATGAAATTGAC TCCAGTGAGATGATGCCAGA TGGTTGTGTACTGTTTTAAGGAAA TGCCAGTAACTTCCCTTTGC CGACAAAATTAAGCTTCTTCGGTA TTTCACTGATTGCAAATTAAATACG TTTGAAACCAGTTCTGATAAGCAT TGCCATATTCAGGTGCCATA TTTAAAAAGATTTATTTCATCTTGCTG GCAGTTAGCAGAATTAACTGAGGA TCTCCCTCTCCATCTCATGC GCATACTATCAGCAATATTGGGTTT TTTTGCAGCAACAAATATGTATTAAA TGTTGGCAAAGATACAACTGAA CCTGAGGTCAAATGCATTCTCT GGGAGTCTCCACTGCAACAT 


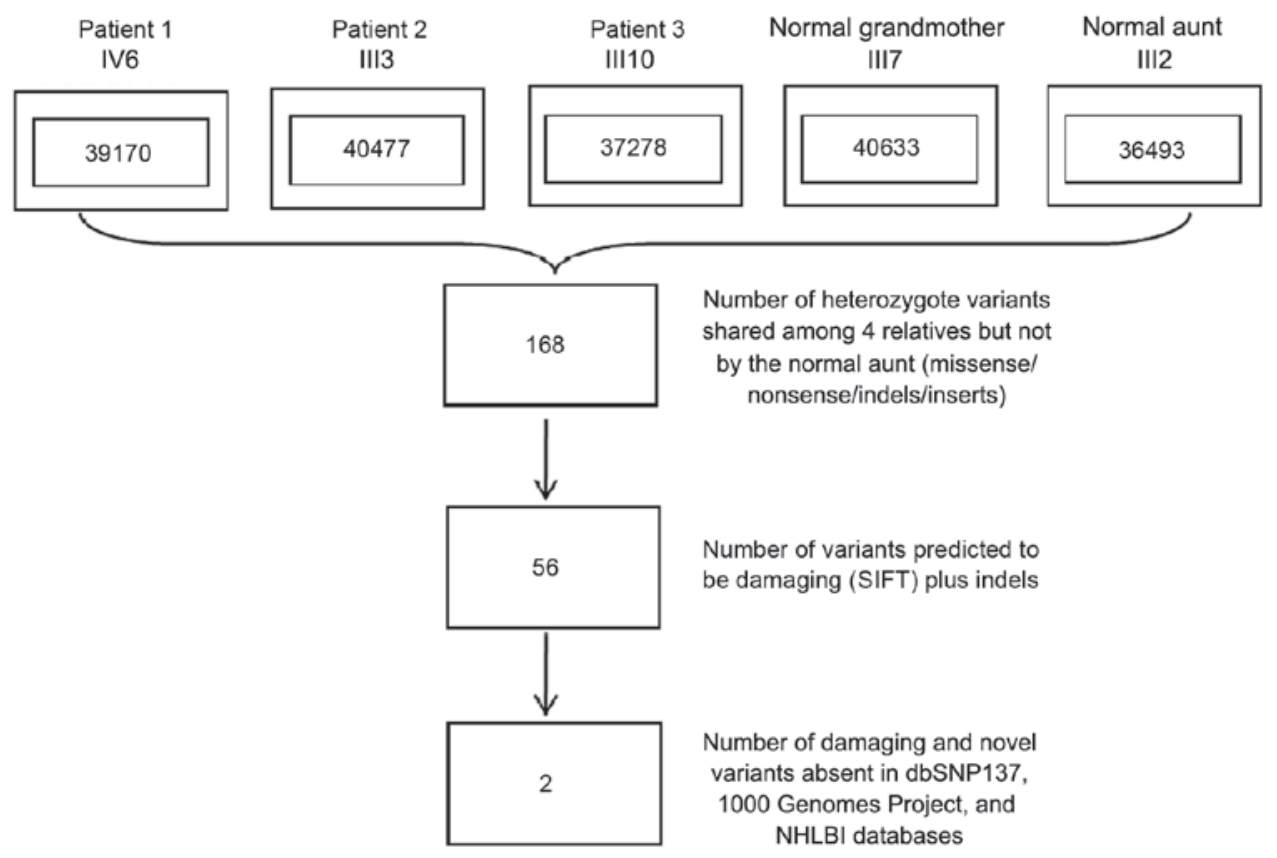

Figure 2. Filtering strategy of susceptibility variants. Numbers of variants are shown in the boxes. SNP, single-nucleotide polymorphism; SIFT, sorting intolerant from tolerant; NHLBI, National Heart, Lung and Blood Institute.

A
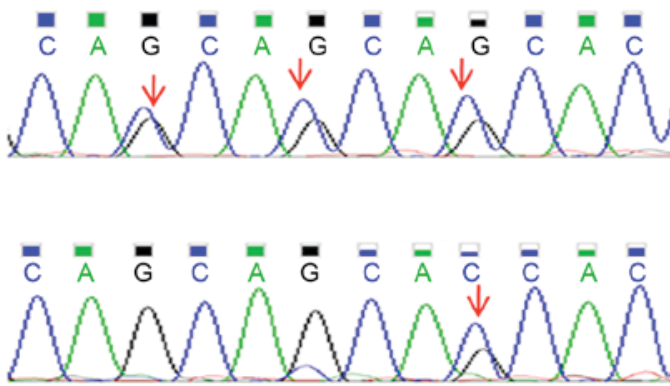

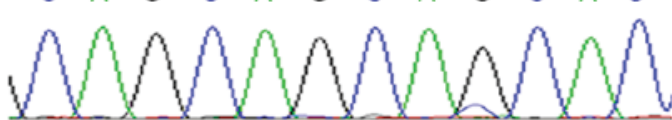

B

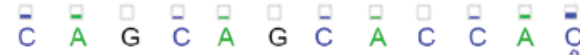

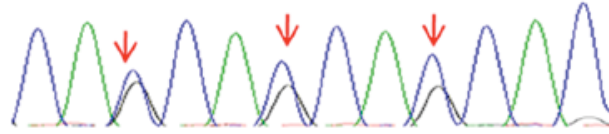

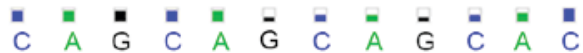
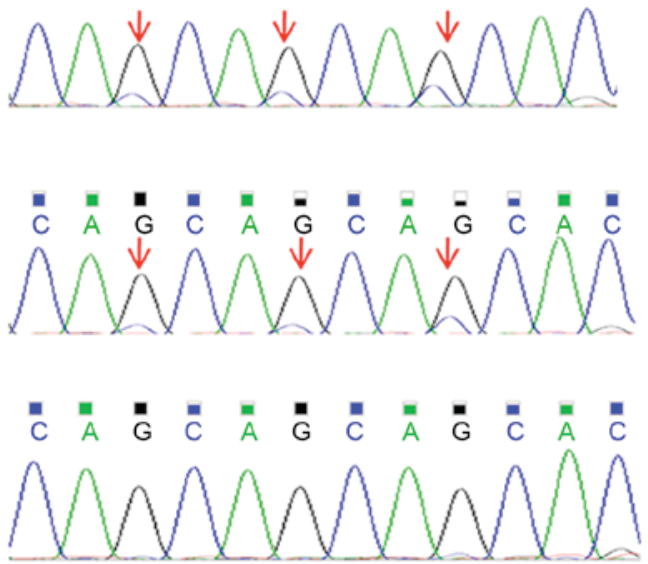

Figure 3. Mutation analysis in subjects with BMP2K mutations. (A) c.1432_1440delCAGCAGCAG and c.1440_1441insCAG mutations in exon 11 of BMP2K in the pedigree as determined by whole-exome sequencing (top one for II7, III10 and IV6 in the pedigree, middle one for III3 in the pedigree, separately) compared to the control (bottom one for III2 in the pedigree). (B) The c.1432_1440delCAGCAGCAG mutation in BMP2K was validated by Sanger sequencing in three unrelated sporadic cases of developmental dysplasia of the hip (top three) compared to one control (bottom one). The mutated nucleotide is indicated by an arrow.

Validation of the presence of the mutations in BMP2K by Sanger sequencing. The presence of CAGCAGCAG/- or -/CAG indels in BMP2K was validated by Sanger sequencing in the four affected individuals, the unaffected related as well as the unaffected unrelated family members. Of the affected individuals, a total of 4/4 (100\%) were positive for the BMP2K variants. The unaffected family members with DNA available (Fig. 1) were also genotyped and 4/15 (26.7\%) of the unaffected related family members and 0/7 (0\%) of the unaffected unrelated (married-in) family members were positive for the variants (Fig. 1). Subsequently, 37 sporadic DDH patients were assessed, three of which were positive for the BMP2K variants (8.12\%) (Fig. 3B). 


\section{Discussion}

DDH has a complex etiology with environmental as well as genetic causes, and appears to be inherited in an autosomal dominant fashion and exhibits incomplete penetrance as described by Feldman et al (11). Previous studies have revealed five loci and a few genes associated with DDH; however, the exact genetic factors associated with a risk of DDH have remained elusive. The present study performed WES and mutational analyses in a large family showing intergenerational inheritance of DDH to identify the genetic basis of the disease. WES analysis, which makes no assumptions regarding the location of a mutation, identified two novel inframe mutations in BMP2K to be the probable disease-causing mutations in a family with DDH in the present study. Sanger sequencing confirmed this result and the mutations were found to be associated with the clinical findings in the three generations with regard to DDH. DDH is a complex disorder with environmental as well as genetic causes and shows incomplete penetrance, and not all individuals who appear unaffected by DDH are free mutations associated with the disease, as was shown in the inheritance of the BMP2K variant in the pedigree. As expected, all affected individuals in the family assessed had either one of these two variants. These two mutations were also present in the DNA of obligate heterozygotes (II2, II7 and II9) even though these individuals did not have any signs of DDH. The validation of these two MNP mutations in 37 unrelated sporadic cases of DDH in the present study appears to show the overrepresentation of the c.1432_1440delCAGCAGCAG mutation. While the present study indicated that the mutations identified represent a considerable genetic risk factor associated with DDH, more sporadic DDH cases and DDH pedigrees require to be tested to determine the prevalence of BMP2K mutations in the overall DDH patient population.

The BMP2K gene is located on chromosome 4q21.21 and was originally identified by Kearns et al (23) as a BMP2-inducible gene. BMPs are multifunctional cytokines belonging to the transforming growth factor- $\beta$ superfamily, comprised of $\sim 50$ genes (24), and were originally identified to have a central role in the normal development of skeletal and osteoblast differentiation during embryonic, fetal and infantile growth periods (25). BMP2 is the most clinically evaluated BMP and has a critical role in early embryogenesis, skeletal development and the differentiation of osteoblasts $(25,26)$. Signaling by BMP2 requires the binding of the BMP2 molecule to the BMP receptors, a set of serine/threonine kinase receptors located on the cell surface of osteoblasts $(27,28)$. The protein encoded by $\mathrm{BMP} 2 \mathrm{~K}$ is a $126-\mathrm{kDa}$ serine/threonine protein kinase containing a nuclear localization signal (23). Protein levels of BMP2K were shown to increase during BMP2-induced differentiation of a mouse osteoblastic cell line, and BMP2K has been identified as an important regulator of the cell differentiation process, suggesting that it is critical for skeletal development and osteoblast differentiation (23). It is therefore plausible that the BMP2K mutations affect the development of the hip.

BMP2K contains a nuclear localization signal to direct protein to nuclei and may affect transcription activities of target genes. It has been identified as a clathrin-coated, vesicle-associated protein, suggesting it may also function to regulate endocytic complexes (29). BMP2K was identified to be an interacting protein of Numb and to participate in the endocytic functions of Numb, which is essential for mammalian development and has a role in regulating the proliferation and differentiation of neural progenitor cell populations during embryogenesis $(30,31)$. It has been reported that signaling pathways associated with Akt, a serine/threonine protein kinase, are involved in osteogenic processes and suppress osteoblast apoptosis (32). Mukherjee and Rotwein (33) found that an intact insulin-like growth factor-induced phosphoinositide-3 kinase/Akt signaling cascade is essential for BMP2-activated osteoblast differentiation and maturation, bone development and growth, and demonstrated that activation of Akt promotes BMP2-mediated osteoblast differentiation. Tseng et al (34) demonstrated that hypoxia induced BMP2 expression via integrin-linked kinase/Akt/mammalian target of rapamycin and hypoxia-inducible factor- $1 \alpha$ pathways in osteoblasts in a time-dependent manner. BMP2K, a serine/threonine kinase protein, may regulate osteoblast differentiation through via Akt-associated signaling pathways. It remains elusive why mutations in BMP2K result in the phenotype of DDH. In addition, whether the BMP2K variants are associated with the risk for DDH remains to be determined. In vitro and in vivo experiments are required to examine the genotype-phenotype correlation.

In recent years, functional studies have shown the importance of BMP2K in the regulation of human osteoblast proliferation and differentiation. Shock wave-stimulated cell proliferation and differentiation, along with induced upregulation of BMP2K gene expression, have been reported in human primary osteoblast cultures (35). Stable expression of BMP2K in MC3T3-E1 osteoprogenitor cells suppressed mature osteoblast function, suggesting the important role of BMP2K in attenuating the program of osteoblast differentiation in mineralized tissue. Furthermore, treatment with 1,25-dihydroxyvitamin $\mathrm{D}$, which is the active form of vitamin $\mathrm{D}$ and has a pivotal role in bone homeostasis and is a potent regulator of osteoblast transcription contributing to fetal and neonatal bone development (36), decreased the levels of BMP2K protein expression in human osteoblasts, indicating that BMP2K negatively regulates human osteoblast proliferation and differentiation (37). These studies suggest that BMP2K is closely associated with the normal development of bone during embryonic, fetal and infantile growth periods. This MNP of c.1432_1440delCAGCAGCAG or c.1440_1441insCAG, corresponding with the Gln478_480del or Gln480ins variations, may cause changes of BMP2K protein activity, affecting bone development and leading to DDH.

In conclusion, the present study proposed two novel polymorphisms in the BMP2K gene, which is known to be associated with skeletal development, and suggested an association of BMP2K with DDH susceptibility in a Han Chinese pedigree and in sporadic DDH cases. While DDH has been previously shown to be associated with genetic factors, the mechanisms have remained largely elusive, and the present study provided a possible mechanism, suggesting that novel BMP2K mutations are implicated in the pathogenesis of DDH. Furthermore, the present study provided important insight into the BMP family as potential signaling molecules in the pathogenesis of DDH. 


\section{Acknowledgements}

The authors are grateful for the participation of the family involved, to whom the present study is dedicated. The present study was supported by the National Natural Science Foundation of China (no. 81401763) the Health and Family Planning Committee of Shanghai Science Foundation (no. 20144Y0176), the Shanghai Science and Technology Committee (no. 12DZ2295006) and '985 Project' Funds from Shanghai Jiaotong University School of Medicine (no. YBKL2013008).

\section{References}

1. Kokavec M and Bialik V: Developmental dysplasia of the hip: Prevention and real incidence. Bratisl Lek Listy 108: 251-254, 2007.

2. Jacobsen S, Sonne-Holm S, Søballe K, Gebuhr P and Lund B Hip dysplasia and osteoarthrosis: A survey of 4151 subjects from the osteoarthrosis substudy of the copenhagen city heart study. Acta Orthop 76: 149-158, 2005.

3. Stein-Zamir C, Volovik I, Rishpon S and Sabi R: Developmental dysplasia of the hip: Risk markers, clinical screening and outcome. Pediatr Int 50: 341-345, 2008.

4. Stevenson DA, Mineau G, Kerber RA, Viskochil DH, Schaefer C and Roach JW: Familial predisposition to developmental dysplasia of the hip. J Pediatr Orthop 29: 463-466, 2009.

5. Czeizel A, Szentpétery J, Tusnády G and Vizkelety T: Two family studies on congenital dislocation of the hip after early orthopaedic screening Hungary. J Med Genet 12: 125-130, 1975.

6. Wynne-Davies R: Acetabular dysplasia and familial joint laxity: Two etiological factors in congenital dislocation of the hip. A review of 589 patients and their families. J Bone Joint Surg Br 52 : 704-716, 1970.

7. Wilkinson JA: Etiologic factors in congenital displacement of the hip and myelodysplasia. Clin Orthop Relat Res: 75-83, 1992.

8. Cilliers HJ and Beighton P: Beukes familial hip dysplasia: An autosomal dominant entity. Am J Med Genet 36: 386-390, 1990.

9. Loughlin J, Mustafa Z, Irven C, Smith A, Carr AJ, Sykes B and Chapman K: Stratification analysis of an osteoarthritis genome screen-suggestive linkage to chromosomes 4, 6, and 16. Am J Hum Genet 65: 1795-1798, 1999.

10. Mabuchi A, Nakamura S, Takatori Y and Ikegawa S: Familial osteoarthritis of the hip joint associated with acetabular dysplasia maps to chromosome 13q. Am J Hum Genet 79 : $163-168,2006$

11. Feldman GJ, Dalsey C, Fertala K, Azimi D, Fortina P, Devoto M, Pacifici $\mathrm{M}$ and Parvizi J: The otto aufranc award: Identification of a $4 \mathrm{Mb}$ region on chromosome 17q21 linked to developmental dysplasia of the hip in one 18-member, multigeneration family. Clin Orthop Relat Res 468: 337-344, 2010.

12. Marschall Y and Distl O: Mapping quantitative trait loci for canine hip dysplasia in German Shepherd dogs. Mamm Genome 18: 861-870, 2007.

13. Rubini M, Cavallaro A, Calzolari E, Bighetti G and Sollazzo V: Exclusion of COL2A1 and VDR as developmental dysplasia of the hip genes. Clin Orthop Relat Res 466: 878-883, 2008.

14. Feldman GJ, Parvizi J, Levenstien M, Scott K, Erickson JA, Fortina P, Devoto M and Peters CL: Developmental dysplasia of the hip: Linkage mapping and whole exome sequencing identify a shared variant in CX3CR1 in all affected members of a large multigeneration family. J Bone Miner Res 28: 2540-2549, 2013.

15. Feldman GJ, Parvizi J, Sawan H, Erickson JA and Peters CL: Linkage mapping and whole exome sequencing identify a shared variant in CX3CR1 in a large multi-generation family. J Arthroplasty 29 (9 Suppl): S238-S241, 2014.

16. Li H and Durbin R: Fast and accurate long-read alignment with Burrows-Wheeler transform. Bioinformatics 26: 589-595, 2010.
17. Plagnol V, Curtis J, Epstein M, Mok KY, Stebbings E, Grigoriadou S, Wood NW, Hambleton S, Burns SO, Thrasher AJ, et al: A robust model for read count data in exome sequencing experiments and implications for copy number variant calling. Bioinformatics 28: 2747-2754, 2012.

18. Wang K, Li M and Hakonarson H: ANNOVAR: Functional annotation of genetic variants from high-throughput sequencing data. Nucleic Acids Res 38: e164, 2010.

19. Cinqolani P, Platts A, Wang le L, Coon M, Nguyen T, Wang L, Land SJ, Lu X and Ruden DM: A program for annotating and predicting the effects of single nucleotide polymorphisms, SnpEff: SNPs in the genome of Drosophila melanogaster strain w1118; iso-2; iso-3. Fly (Austin) 6: 80-92, 2012.

20. Kumar P, Henikoff S and Ng PC: Predicting the effects of coding non-synonymous variants on protein function using the SIFT algorithm. Nat Protoc 4: 1073-1081, 2009.

21. Adzhubei IA, Schmidt S, Peshkin L, Ramensky VE, Gerasimova A, Bork P, Kondrashov AS and Sunyaev SR: A method and server for predicting damaging missense mutations. Nat Methods 7: 248-249, 2010.

22. Schwarz JM, Rödelsperger C, Schuelke M and Seelow D: Mutation taster evaluates disease-causing potential of sequence alterations. Nat Methods 7: 575-576, 2010

23. Kearns AE, Donohue MM, Sanyal B and Demay MB: Cloning and characterization of a novel protein kinase that impairs osteoblast differentiation in vitro. J Biol Chem 276: 42213-42218, 2001

24. Chen G, Deng C and Li YP: TGF- $\beta$ and BMP signaling in osteoblast differentiation and bone formation. Int J Biol Sci 8: 272-288, 2012

25. Li X and Cao X: BMP signaling and skeletogenesis. Ann N Y Acad Sci 1068: 26-40, 2006.

26. Kanzler B, Foreman RK, Labosky PA and Mallo M: BMP signaling is essential for development of skeletogenic and neurogenic cranial neural crest. Development 127: 1095-1104, 2000.

27. Nakamura Y, Wakitani S, Nakayama J, Wakabayashi S, Horiuchi $\mathrm{H}$ and Takaoka K: Temporal and spatial expression profiles of BMP receptors and noggin during BMP-2-induced ectopic bone formation. J Bone Miner Res 18: 1854-1862, 2003.

28. Ishidou Y, Kitajima I, Obama H, Maruyama I, Murata F, Imamura T, Yamada N, ten Dijke P, Miyazono K and Sakou T: Enhanced expression of type I receptors for bone morphogenetic proteins during bone formation. J Bone Miner Res 10: 1651-1659, 1995.

29. Borner GH, Antrobus R, Hirst J, Bhumbra GS, Kozik P, Jackson LP, Sahlender DA and Robinson MS: Multivariate proteomic profiling identifies novel accessory proteins of coated vesicles. J Cell Biol 197: 141-160, 2012.

30. Krieger JR, Taylor P, Gajadhar AS, Guha A, Moran MF and McGlade CJ: Identification and selected reaction monitoring (SRM) quantification of endocytosis factors associated with Numb. Mol Cell Proteomics 12: 499-514, 2013.

31. Petersen PH, Zou K, Hwang JK, Jan YN and Zhong W: Progenitor cell maintenance requires numb and numblike during mouse neurogenesis. Nature 419: 929-934, 2002.

32. Choi YH, Jeong HM, Jin YH, Li H, Yeo CY and Lee KY: Akt phosphorylates and regulates the osteogenic activity of Osterix. Biochem Biophys Res Commun 411: 637-641, 2011.

33. Mukherjee A and Rotwein P: Akt promotes BMP2-mediated osteoblast differentiation and bone development. J Cell Sci1 22: 716-726, 2009

34. Tseng WP, Yang SN, Lai $\mathrm{CH}$ and Tang $\mathrm{CH}$ : Hypoxia induces BMP-2 expression via ILK, Akt, mTOR, and HIF-1 pathways in osteoblasts. J Cell Physiol 223: 810-818, 2010.

35. Hofmann A, Ritz U, Hessmann MH, Alini M, Rommens PM and Rompe JD: Extracorporeal shock wave-mediated changes in proliferation, differentiation, and gene expression of human osteoblasts. J Trauma 65: 1402-1410, 2008

36. Goltzman D, Hendy GN and White JH: Vitamin D and its receptor during late development. Biochim Biophys Acta 1849: 171-180, 2015.

37. Lisse TS, Chun RF, Rieger S, Adams JS and Hewison M: Vitamin D activation of functionally distinct regulatory miRNAs in primary human osteoblasts. J Bone Miner Res 28: 1478-1488, 2013. 